\title{
Coherent reflected pulses of exciton polaritons in multiple quantum wells at Brewster's-angle incidence
}

\author{
Byung-Ryool Hyun, Tomobumi Mishina, and Yasuaki Masumoto \\ Institute of Physics, University of Tsukuba, Tsukuba, Ibaraki 305, Japan \\ Masaaki Nakayama \\ Department of Applied Physics, Faculty of Engineering, Osaka City University, Sugimoto, Sumiyoshi-ku, Osaka 558, Japan
}

(Received 2 September 1997)

\begin{abstract}
A coherent emission of excitons in semiconductor multiple quantum wells was investigated by using transient Brewster reflection spectroscopy. The method reduced nonresonant background reflection greatly and allowed us to observe the coherent dynamics of the excitons clearly. The coherent signal showed not only the quantum beats of the heavy- and light-hole excitons but also the oscillatory modulation structures that resulted from the radiative coupling of exciton polaritons. In the linear regime, these observations were compared with results calculated from the transfer-matrix method involving multiple-reflection effects, and good agreement was obtained. As the excitation density of the incident pulses was raised, the decay rate of the coherent signal increased due to the exciton-exciton scattering.
\end{abstract}

[S0163-1829(97)52244-2]

\section{INTRODUCTION}

The investigation of coherent phenomena of exciton systems has become an active and fast progressing field of research. The radiative properties and propagation effects of excitonic polaritons in bulk semiconductors and quantum wells especially have been of considerable interest in fundamental studies.

The behaviors of exciton polaritons on the temporal shape of ultrashort laser pulses have been investigated mainly in the transmission geometry. If the input pulse bandwidth is wider than the exciton resonance linewidth, the temporal shape of the transmitted pulse is adjusted into the severely distorted and aperiodic modulation due to the interference between polaritons of the upper and lower branches. ${ }^{1-4}$

Another simple and useful way to study the coherent dynamics of exciton polaritons is the transient reflection spectroscopy which up to now was not often used. This measurement enables us to investigate the optical proprieties of the as-grown sample without removal of the substrate. Moreover, the use of the Brewster's-angle condition can reduce the nonresonant background reflection greatly and give us a clear time-resolved signal of the exciton with the improved signal-to-noise ratio. ${ }^{5,6}$

In this paper we report the oscillatory free induction decay (FID) signal in the excitation of the single resonance line and quantum beats between the heavy-hole and light-hole excitons superimposed on the FID signal. We evaluated the time evolution of the reflected pulse at the Brewster's-angle condition by using the transfer-matrix method in the linear regime. The experimental results were well reproduced by the numerical calculation. The decay rate of the coherent signal increases with the excitation density due to the scattering of excitons.

\section{EXPERIMENT}

The sample used in the present study was a high-quality multiple quantum well (MQW) structure consisting of 25 periods of $(\mathrm{GaAs})_{53} /(\mathrm{AlAs})_{53}$ grown by molecular-beam epitaxy on a (001)-oriented semi-insulating GaAs substrate. Figure 1 shows the reflection spectrum at the Brewster'sangle condition. The lowest $n=1$ heavy- and light-hole exciton transitions of the MQW structure were observed at 1.538 and $1.546 \mathrm{eV}$, respectively. The sample was mounted in a liquid-helium cryostat and cooled to $2 \mathrm{~K}$ during all the experiments.

For a light source of time-resolved experiments, a selfmode-locked Ti:sapphire laser producing $130 \mathrm{fs}$ pulses at an $82 \mathrm{MHz}$ repetition rate was used. Our experiments were performed with a fast-scan setup, which was equipped with a shaker instead of a scanning optical delay for varying the time delay. We took the time trace of the Brewster-reflected optical pulses by using a standard up-conversion method. The up-converted signal was detected by a photomultiplier tube and recorded by a digital accumulator. This real-time data acquisition significantly improved the signal-to-noise ratio.

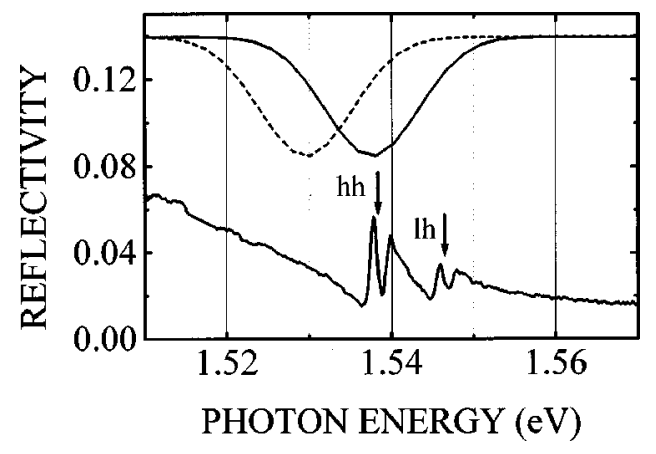

FIG. 1. The reflection spectrum of a GaAs MQW sample at Brewster's-angle incidence. The lowest $n=1$ heavy-hole and lighthole exciton transitions occur at 1.538 and $1.546 \mathrm{eV}$, and are indicated by arrows. The energy splitting of two exciton resonances is 8 $\mathrm{meV}$. The solid and dotted lines are spectra of the femtosecond laser pulses used in the time-resolved experiments. 


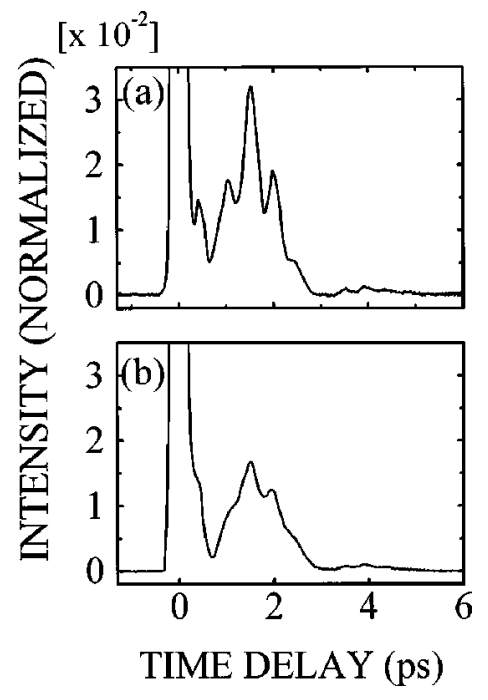

FIG. 2. The time-resolved reflected pulse signals in GaAs MQWS (a) on resonance with the $1 s$ heavy-hole exciton and (b) at detuning of $7.1 \mathrm{meV}$ below the $1 \mathrm{~s}$-hh exciton.

\section{RESULTS AND DISCUSSIONS}

Figure 2 shows the temporal evolution of the Brewsterreflected pulse in the linear regime $\left(17 \mathrm{~nJ} / \mathrm{cm}^{2}\right)$. The timeresolved signals have a strong peak at zero delay, corresponding to the reflected laser pulse from the sample surface. The following delayed tail comes from the coherent emission of excitons. The coherent tail shows a slowly oscillatory modulation and a clear beating structure superimposed on it. The period of beat (500 fs) corresponds to the energy difference of $8 \mathrm{meV}$. It is consistent with the energy splitting between two transition lines in the reflection spectrum. This structure of the quantum beats resulted from the interference between the heavy-hole and the light-hole exciton transitions. Figures 2(a) and 2(b) are the results corresponding to the laser spectrum profiles in Fig. 1, respectively. Since Fig. 2 (a) is the emission signal under the simultaneous excitation of the heavy-hole and light-hole exciton resonances, the quantum beat on the oscillatory modulation is seen more clearly.

In the case of Fig. 2(b), the coherent signal also clearly exhibited a slowly oscillatory modulation signal and the small beating structure superimposed on the signal. The reduction of the quantum beats implies the small contribution of the light-hole exciton because the spectrum overlap of the laser pulse and the light-hole exciton becomes small. Therefore we can conclude that the slowly oscillatory modulation is originated from the single heavy-hole exciton transition.

It has already been reported that the ultrashort pulse propagation around the single resonance line of the bulk semiconductors leads to the oscillatory modulation in the transmitted pulse. ${ }^{1,2}$ It originated from the interference between polaritons of the upper and lower dispersion branches.

Contrary to the polariton states in the bulk semiconductors, in quantum wells the breaking of translational invariance along one direction gives the exciton state an intrinsic radiative decay mechanism which emits photons. This radiative decay mechanism couples the exciton states in different wells. In quantum wells coupled by the light field, the collective effect can take place. The collective effects consist of the electromagnetic coupling between excitons located in different wells, and it exists even if the wells are electronically uncoupled. As a result, the coupled excitons are formed and distributed throughout the whole well in the MQW. This radiative coupling is attributed to the physical origin of our experimental results.

Therefore, the transient behavior of the reflected signal can be understood in the exciton-polariton scheme. It is well known that the group velocity of exciton-polariton branches has a minimum velocity at the exciton resonance due to polariton dispersion. Near the exciton resonance region upper and lower branches of exciton polaritons have different group velocities which lead to relative phase difference. ${ }^{7,8}$ As a consequence, during the propagation of the ultrashort pulses, the superposition of these reflected pulses results in beating structures. Hence, the slowly oscillatory modulation structure could be considered a result of the radiative coupling effect of the exciton polaritons distributed in the MQW while the ultrashort pulses propagate.

It is noteworthy that in recent theoretical investigations collective effects can modify the transmission and reflection spectra in MQW structures and become important for the excitonic decay process. ${ }^{9,10}$

In order to quantitatively understand the experimental result, we calculated the reflected pulse shape by using a transfer matrix. The reflected pulse amplitude $E_{R}(t)$ is given by

$$
E_{R}(t, \theta)=\frac{1}{2 \pi} \int E_{I}(\omega) r(\omega, \theta) e^{i \omega t} d \omega
$$

where $E_{I}(\omega)$ is the Fourier transform of the incident electric field. $r(\omega, \theta)$ is the frequency- and angle-dependent reflection coefficient of the sample where $\theta$ is the incident angle. The reflection coefficient for the $p$-polarized electric field can be obtained by the transfer-matrix method. ${ }^{11}$ The transfer matrix of the whole MQW is obtained by multiplying the transfer matrices of each layer and of each interface. Therefore, the transfer-matrix method automatically takes into account the effects related to multiple reflections which play an important role in the MQW structures. ${ }^{12,13}$ For the linear regime this method can reveal characteristic features of the reflected pulse shape in the MQW sample.

The excitonic contribution to the effective local dielectric function of each layer is expressed as a Lorentizian oscillator model,

$$
\epsilon(\omega)=\epsilon_{b}\left[1+\sum_{\sigma} \frac{\omega_{\mathrm{LT}}^{\sigma}}{\omega_{0}^{\sigma}-\omega-i \gamma^{\sigma}}\right]
$$

where $\epsilon_{b}$ is the background dielectric constant. $\omega_{\mathrm{LT}}^{\sigma}, \omega_{0}^{\sigma}$, and $\gamma^{\sigma}$ are the longitudinal-transverse (LT) splitting, the resonant frequency, and the damping factor of each exciton in the layer, respectively. The LT splitting of the barrier and substrate was assumed to be zero (no excitons effect) and the exciton parameters of the quantum well were taken as $\omega_{\mathrm{LT}}^{\mathrm{hh}}=0.6, \omega_{\mathrm{LT}}^{\mathrm{lh}}=0.4, \gamma^{\mathrm{hh}}=0.13$, and $\gamma^{\mathrm{lh}}=0.4 \mathrm{meV} . \epsilon_{b}$ was taken as the background dielectric constant of each bulk semiconductor. ${ }^{14}$

The $j$ th layer is represented by the $2 \times 2$ transfer matrix 


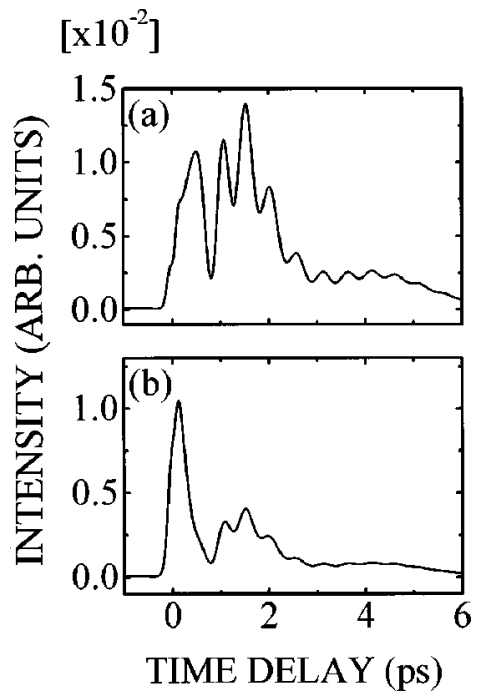

FIG. 3. The calculated results of the reflected pulses in the Brewster's-angle incidence by the transfer-matrix method. (a) The central frequency of the laser pulse is $1.538 \mathrm{eV}$ (heavy-hole exciton). (b) The central frequency of the laser pulses is changed into $1.5309 \mathrm{eV}$.

$$
M_{j}=\left(\begin{array}{cc}
\cos k_{j} d_{j} & -i \sin k_{j} d_{j} / Z_{j} \\
-i Z_{j} \sin k_{j} d_{j} & \cos k_{j} d_{j}
\end{array}\right),
$$

where $d_{j}$ is the thickness of the $j$ th layer, and $Z_{j}(\omega, \theta)$ and $k_{j}(\omega, \theta)$ are the impedance and wave number of the respective medium as defined by

$$
Z_{j}(\omega, \theta)=\cos \theta_{j} / n_{j}(\omega), \quad k_{j}(\omega, \theta)=\frac{c}{\omega} Z_{j} \cos \theta_{j}
$$

where $n_{j}(\omega)\left(=\sqrt{\epsilon(\omega) / \epsilon_{0}}\right)$ is the complex refractive index, and $\theta_{j}$ and $\epsilon_{0}$ are the incident angle in the medium and the permittivity of a vacuum, respectively.

If the resultant matrix for the MQW structure is $T_{R}=\left(t_{i j}\right)$, we can obtain the complex reflection coefficient,

$$
r(\omega, \theta)=\frac{Z_{\text {in }}\left(t_{11}+Z_{\text {out }} t_{12}\right)-\left(t_{21}+Z_{\text {out }} t_{22}\right)}{Z_{\text {in }}\left(t_{11}+Z_{\text {out }} t_{12}\right)+\left(t_{21}+Z_{\text {out }} t_{22}\right)},
$$

where $Z_{\text {in }}$ and $Z_{\text {out }}$ are the wave impedance of the entrance and exit regions.

Figure 3 shows the calculated results for the temporal shape of the reflected pulse in the MQW structure under the Brewster's-angle condition. Figure 3(a) corresponds to the laser tuning on the heavy-hole exciton resonance (the solid laser profile in Fig. 1). We can see that the calculation describes fairly well the quantitative features of experimental results, a slowly oscillatory behavior of FID and quantum beats with $500 \mathrm{fs}$ period.

When the central frequency of the incident electric field was detuned from the $1 \mathrm{~s}$ heavy-hole exciton resonance by $7.1 \mathrm{meV}$ corresponding to the dashed laser profile in Fig. 1, the reduction of the oscillatory modulation and the small beating structure on the modulation signal was reproduced in Fig. 3(b). We clearly show the oscillatory modulation signal. In the fitting procedures, the oscillatory modulation behavior was strongly dependent on the parameters of the heavy-hole

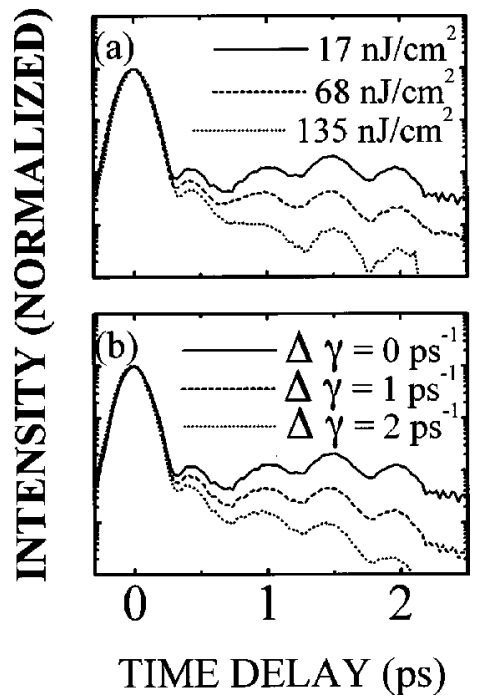

FIG. 4. The excitation density dependence of the reflected pulse at the Brewster's-angle condition. (a) As the excitation density is raised, the decay of the experimental coherent signal becomes faster due to the exciton-exciton scattering. (b) The fitting results obtained by the equation $I=I_{0}(t) \exp (-\Delta \gamma t) . I_{0}(t)$ is the experimental signal for the lowest excitation density and $\Delta \gamma\left(\mathrm{ps}^{-} 1\right)$ is the scattering rate. The fitting shows a good correspondence with the experimental results of (a).

exciton. From the calculated results, we could confirm that the slowly oscillatory modulation originates from the only heavy-hole exciton resonance.

It was noteworthy that in Fig. 3 the relatively small peak at zero delay compared to the experimental results was attributed to the experimental inaccuracy of the Brewster'sangle condition which increases the nonresonant background reflection in the time-domain signal. Even a small angle deviation $\left(\sim 1^{\circ}\right.$ or $\left.2^{\circ}\right)$ made the peak at zero delay strongly increase while the intensity of the coherent emission signal was not changed. Therefore the relatively strong peak at zero delay in the experimental data is probably attributed to the small angle deviation from the exact Brewster's angle and the scattering on the sample surface.

In this way, our experimental result was well explained by a simple calculation method in the exciton-polariton pictures. In our calculation, we neglected the spatial dispersion coming from the finite exciton mass. In the QW case, the kinetic energy of the exciton at $K_{\|}$is six orders of magnitude smaller than the damping factor of each exciton. Therefore the kinetic energy of the exciton has a very small contribution in the few picosecond region. As can be seen, we nicely reproduced the experimental results by a simple method without considering the spatial dispersion effects.

We have also investigated the excitation density dependence of the time evolution of the reflected pulse, when the laser is tuned to the heavy-hole exciton transition. Figure 4(a) shows the temporal shapes of the reflected pulse intensity for the three excitation densities. A clear quantum beat modulation is observed for all the excitation densities. As the excitation density is raised, the decay of the coherent emission becomes faster.

The fitting of the excitation density dependence is plotted in Fig. 4(b). The time trace for the case $\Delta \gamma=0 \mathrm{ps}^{-1}$ is the 
experimental result for $17 \mathrm{~nJ} / \mathrm{cm}^{2}$ excitation density and represents a linear response signal. The trace for the case of $\Delta \gamma=1 \mathrm{ps}^{-1}$ and $2 \mathrm{ps}^{-1}$ are obtained by multiplying the timedependent coefficient $\exp (-\Delta \gamma t)$ by the linear signal. The fitting shows a good correspondence with the experimental results of Fig. 4(a). In spite of the complex nature of the coherent signal, the fitting result indicates that the excitation dependence has a linear relationship with a single parameter, $\Delta \gamma$.

It was known theoretically and experimentally that the homogeneous linewidth of excitons in GaAs quantum wells, which is proportional to the inverse phase-coherence time $T_{2}^{-1}$, has a linear dependence on the density. ${ }^{15-17}$ The agreement between the experimental and the theoretical line and the linear correspondence between the excitation density and the broadening constant (collision efficiency) show that the line broadening is due to the exciton-exciton scattering model.

\section{CONCLUSIONS}

We have observed the coherent emission signal from the excitons in GaAs/AlAs MQW structures by the timeresolved Brewster reflection experiment. The reflected signal showed the oscillatory behavior caused by the radiative coupling of exciton polaritons in a sample of nonzero size. The quantum beats of the hh and lh exciton were clearly observed. The period of beats (500 fs) corresponds to an energy difference between the two exciton transition lines. The calculation based on the transfer matrix method considering multiple reflections reproduced the experimental features very well. The decay rate of the coherent signal increases with the excitation density due to the exciton-exciton collision.
${ }^{1}$ J. E. Rothenberg, D. Grischkowsky, and A. C. Balant, Phys. Rev. Lett. 53, 552 (1984).

${ }^{2}$ D. Fröhlich, A. Kulik, B. Uebbing, A. Mysyrowicz, V. Langer, H. Stolz, and W. von der Osten, Phys. Rev. Lett. 67, 2343 (1991).

${ }^{3}$ T. Mishina and Y. Masumoto, Phys. Rev. Lett. 71, 2785 (1993).

${ }^{4}$ D. S. Kim, J. Shah, D. A. B. Miller, T. C. Damen, A. Vinattieri, W. Schäfer, and L. N. Pfeiffer, Phys. Rev. B 50, 18240 (1994).

${ }^{5}$ J. Aaviksoo and J. Kuhl, IEEE J. Quantum Electron. 25, 2523 (1989).

${ }^{6}$ T. Kuroda, K. Inoue, I. Suemune, and R. Kuribayashi, in Proceedings of the 22nd International Conference on the Physics of Semiconductors, edited by D. J. Lockwood (World Scientific, Singapore, 1995).

${ }^{7}$ Y. Masumoto, Y. Unuma, Y. Tanaka, and S. Shionoya, J. Phys. Soc. Jpn. 47, 1844 (1979).

${ }^{8}$ R. G. Ulbrich and G. W. Fehrenbach, Phys. Rev. Lett. 43, 963 (1979).

${ }^{9}$ T. Stroucken, A. Knorr, C. Anthony, A. Schulze, P. Thomas, S.
W. Koch, M. Koch, S. T. Cundif, J. Feldmann, and E. O. Göbel, Phys. Rev. Lett. 74, 2391 (1995).

${ }^{10}$ T. Stroucken, A. Knorr, P. Thomas, and S. W. Koch, Phys. Rev. B 53, 2026 (1996).

${ }^{11}$ M. Born and E. Wolf, Principles of Optics (Pergamon, New York, 1970), Secs. 1.5 and 1.6.

${ }^{12}$ G. Manzke and K. Henneberger, Phys. Status Solidi B 147, 733 (1988).

${ }^{13}$ Y. Chen, F. Bassani, J. Massies, C. Deparis, and G. Neu et al., Europhys. Lett. 14, 483 (1991).

${ }^{14}$ R. Harel, E. Cohen, E. Linder, Arza Ron, and L. N. Pfeiffer, Phys. Rev. B 53, 7868 (1996).

${ }^{15}$ S. Schmitt-Rink, D. S. Chemla, and D. A. B. Miller, Phys. Rev. B 32, 6601 (1985).

${ }^{16}$ G. Manzke, K. Henneberger, and V. May, Phys. Status Solidi B 139, 233 (1987).

${ }^{17}$ A. Honold, L. Schultheis, J. Kuhl, and C. W. Tu, Phys. Rev. B 40, 6442 (1989). 